\title{
Prevalence and correlates of loneliness in India: A systematic review
}

\author{
Md Mahbub Hossain*, ${ }^{1}$ Neetu Purohit, ${ }^{2}$ Nusrat Khan, ${ }^{3}$ E. Lisako J. McKyer, ${ }^{1}$ Ping Ma, ${ }^{1}$ \\ Sudip Bhattacharya, ${ }^{4}$ Priyanka Pawar ${ }^{5}$
}

\begin{abstract}
Introduction: Loneliness is a critical psychosocial problem that affects mental health and wellbeing of the individuals and communities. This systematic review synthesized the current evidence on the prevalence and correlates of loneliness from empirical studies conducted in India.

Methods: Adopting the Preferred Reporting Items for Systematic Reviews and Meta-Analysis (PRISMA) guidelines, eight major databases and additional sources were searched. Studies were evaluated using following criteria: peer-reviewed journal articles, published in English language, presenting any quantitative form of evidence on loneliness, and studies conducted in India among the Indian population. A meta-analysis of the prevalence studies and narrative synthesis of the overall status and factors associated with loneliness were conducted.

Results: Among 1290 studies, only 13 met our criteria. All the studies reported a high burden of loneliness among the participants. The pooled prevalence among 3169 participants was $41 \%$ (95\% confidence interval [CI] $18 \%-63 \%$ ). The burden of loneliness was higher among the elderly participants $(44 \%, 95 \%$ CI $5 \%$ - 83\%) compared to younger participants $(24 \%, 95 \%$ CI $22 \%$ $27 \%$ ). Factors associated with loneliness included aging, family structure, marital status, religious practices, group membership, educational attainment, source and level of income, psychological attributes, and comorbid physical and mental health conditions among the affected individuals.

Conclusion: This review identified and evaluated the status and severity of loneliness and its correlates, which may inform future studies examining complex psychopathological and social dynamics associated with loneliness. Lastly, the current evidence necessitates the development of multipronged interventions to address the risk factors and alleviate the burden of loneliness in India.
\end{abstract}

Keywords: Loneliness; Social Isolation; Systematic review; Meta-analysis; India

\footnotetext{
${ }^{1}$ Department of Health Promotion and Community Health Sciences, School of Public Health, Texas A\&M University, TX 77843, USA.

${ }^{2}$ The IIHMR University, Jaipur, Rajasthan 302029, India.

${ }^{3}$ Department of Public Health and Primary Care, University of Cambridge, UK.

${ }^{4}$ Department of Community Medicine, Himalayan Institute of Medical Sciences, Dehradun, Uttarakhand, India.

${ }^{5}$ Mamta Health Institute for Mother and Child, New Delhi 110048, India.

Corresponding author: Md Mahbub Hossain, Texas A\&M School of Public Health, College Station, TX 77843, USA.

Email: mhossain@tamu.edu
} 


\section{Introduction}

Loneliness is one of the fastest-growing psychosocial health concerns in the modern age (Cacioppo and Cacioppo, 2018; Rubin, 2017). The concept of loneliness is explored across various academic disciplines; including psychiatry, psychology, and social work (Hawkley and Cacioppo, 2010; Mushtaq et al., 2014). From existential, psychopathological, and sociological perspectives, loneliness is conceptualized as a depressive state of mind in which the affected individuals may have an internal and unpleasant subjective experience along with qualitative or quantitative loss in the social networks (Bandari et al., 2019; De Jong Gierveld et al., 2015). Moreover, loneliness is considered different than being or living alone (Bandari et al., 2019). Scholars have argued that individuals may suffer from loneliness even in the presence of people around themselves (Bandari et al., 2019; Hawkley and Cacioppo, 2010). In recent years, the severity of loneliness has been reported across many populations (Beutel et al., 2017; Lee et al., 2018; Mullen et al., 2019). A study conducted among community-dwelling adults in California found the prevalence of moderate-high level of loneliness as $76 \%$ (Lee et al., 2018). Another study found the $26.2 \%$ of older adults attending primary care in China were suffering from loneliness (Zhong et al., 2018). Moreover, people living with developmental or intellectual disabilities may have a high burden of loneliness (Alexandra et al., 2018). A high burden of loneliness was also found among military veterans (Wilson et al., 2018), highlighting the magnitude of the problem among diverse occupational groups. Also, many studies evaluated numerous factors associated with loneliness among different population groups including older age, impaired interpersonal and family relationships, low educational attainment, financial hardship, personality traits, low self-efficacy beliefs, physical and mental co-morbidities, social engagement, group membership, and sociocultural attributes (Levy et al., 2017; Yang and Gu, 2019). Furthermore, a number of studies have evaluated the health impacts of loneliness and how it influences the physical, mental, and social wellbeing of the individuals. People living with loneliness experience a higher burden of depressive illness, impaired cognitive functions, dementia, Alzheimer disease, hypertension, and cardiovascular diseases (Lee et al., 2018; Mushtaq et al., 2014; Rafnsson et al., 2017). Moreover, the adverse health effects of loneliness and social disconnection are compared to smoking 15 cigarettes per day (Holt-Lunstad et al., 2010). In addition, loneliness is found to be associated with a $26 \%$ increase in the risk of premature mortality (Holt-Lunstad et al., 2015). This evidence 
suggests loneliness can affect people of different ages, conditions, professions, and places- making it one of the widespread public health challenges to be addressed in our time.

While most of the studies reporting the burden of loneliness were conducted in the industrialized nations where aging and many socioeconomic stressors are affecting social networks, similar problems are emerging in low- and middle- income countries (LMICs) like India (Grover, 2019). Demographic and epidemiological transitions in India have resulted in a decline in the burden of infectious diseases, increased life expectancy at birth, higher proportion of the aging population compared to the past, and a growing burden of chronic conditions (Nethan et al., 2017). In addition, socioeconomic challenges like high poverty, income inequality, low education, high dependency ratio, lack of transportation, unplanned urbanization, rapid industrialization, and a deterioration in social capital have been affecting the overall wellbeing and increasing the likelihood of loneliness among the Indian population (Tiwari, 2013). A nationwide survey conducted in 2004 reported that more than 1.23 million men and 3.68 million women were living alone in India (Jeyalakshmi et al., 2011). Despite a high burden of loneliness across the nation, there is a lack of empirical evidence on the prevalence or other quantitative measures of the severity of loneliness in India. Additionally, it is critical to understand how different sociodemographic factors, socio cultural norms and co-morbidities are associated with loneliness to inform further research and interventions to address the same. This systematic review aimed to address these knowledge gaps. The primary objective of this review was to synthesize the current evidence on the severity of loneliness expressed as prevalence, proportion, level, intensity, or other quantitative measures across studies conducted in India. Secondarily, we aimed to evaluate the sociodemographic factors and psychosocial conditions associated with loneliness as reported in those empirical studies.

\section{Methods}

\section{Study design and search strategy}

This systematic review was conducted in accordance with the Preferred Reporting Items for Systematic Reviews and Meta-Analysis (PRISMA) guidelines (Liberati et al., 2009). Earlier systematic reviews on loneliness in other countries or populations have shown varying measures of studying the severity and associated factors of loneliness among the included studies (Alexandra 
et al., 2018; Wilson et al., 2018). In this systematic review, we considered the prevalence, incidence, rate, proportion, level, or any other quantitative measures which informed the severity of loneliness in India. Moreover, we considered both the sociodemographic and clinical correlates to understand the factors associated with loneliness. To retrieve the scientific evidence on loneliness in the context of India, we developed a search strategy with the following keywords with "OR" Boolean operator to retrieve all available literature on loneliness: "lonely", "loneliness", "social connect*". Further, we used "India" with "AND" operator combining with the earlier string to retrieve studies conducted in India. These keywords were used both as subject headings and general keywords for searching MEDLINE, EMBASE, PsycINFO, ERIC, CINAHL, Academic Source Ultimate, Health Policy Reference Center, and Scopus databases. All databases were searched from their inception to August 31, 2019. Moreover, we included articles from additional sources, including hand-searching, reaching subject experts, and reference searching. All the citations retrieved from databases and additional sources were screened using Rayyan QCRI and RefWorks tools, which are used for systematic screening and citations management, respectively.

\section{Study eligibility: inclusion and exclusion criteria}

While screening the retrieved citations, we included a citation if it was: a) an empirical study, b) conducted in India, c) reported the severity of loneliness at the population level in any of the quantitative measures (including prevalence, incidence, rate, proportion, or level), d) published as an article in a peer-reviewed journal, and e) the language of the publication was English. In this screening process, we excluded citations which were: a) non-empirical in nature (including letters, editorials, reviews, commentaries, opinions) b) conducted outside India, c) did not report any quantitative measure of loneliness among the study participants, d) were not articles published in peer-reviewed journals (including dissertations, reports, policy briefs), and e) not published in English language.

\section{Data extraction and synthesis}

Two reviewers independently reviewed and screened the retrieved literature. At the end of the primary screening, conflicts regarding the eligibility of the studies were resolved in the presence of a third author based on discussion and consensus. Further, full texts of the preliminarily included citations were retrieved and evaluated. After full-text evaluation, studies which met all the inclusion and exclusion criteria were recruited in this systematic review. A pre-designed codebook 
was used to collect data on the location of the study, publication year, study design and instruments, sample size, sample characteristics, recruitment strategy, the severity of loneliness as expressed quantitatively, sociodemographic factors and clinical conditions associated with loneliness as reported in the empirical studies. Two authors independently extracted data and resolved the conflicts through consensus at the end of the extraction process. Further, we conducted a meta-analysis of the prevalence studies to retrieve the pooled prevalence of loneliness using Der-Simonian and Laird's random-effects model with 95\% confidence interval (CI) (DerSimonian and Laird, 1986). In this analysis, positive cases were divided by the total number of samples using intercept-only random effects logistic regression model fitted to obtain the pooled estimate of prevalence. To estimate the prevalence considering the impacts of different variables like age groups or types of the instruments, we conducted subgroup analysis to estimate the pooled prevalence as per those attributes across studies. We used "metaprop_one" program in Stata 15.0 software (College Station, TX), which provided estimates without excluding 0\% or 100\% proportions. In addition, this process reported study-specific and pooled confidence intervals within admissible values, $I^{2}$ statistics enabling interpretation of the heterogeneity of the studies, and $p$-value from $Q$-statistics to evaluate the between-study variability. Furthermore, we narratively synthesized remaining data on severity of and factors associated with loneliness due to low number of articles and a high heterogeneity across studies in terms of methods, populations, and measurements.

\section{Quality assessment of the recruited studies}

We used the National Heart, Lung, and Blood Institute (NHLBI) Study Quality Assessment Tool for observational cohort and cross-sectional studies to assess the methodological quality of the studies included in this systematic review.(National Heart Lung and Blood Institute (NHLBI), 2018) The NHLBI tool consisted of 14 items where each item is assessed through as a question with possible answers as "yes", "no", or other (cannot determine, not applicable, or not reported). The NHLBI tool facilitated scoring and weighing the evidence to rate the overall quality of each study as 'good', 'fair', or 'poor' indicating the validity of the findings in the respective stud 


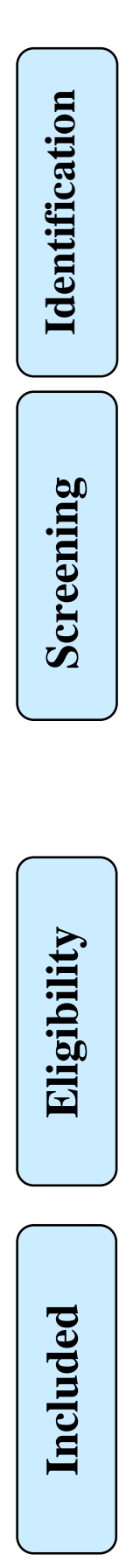

Records identified through searching MEDLINE, EMBASE, PsycINFO, ERIC, CINAHL, Academic Search Ultimate, Health Policy Reference Center, and Scopus databases $(n=1026)$

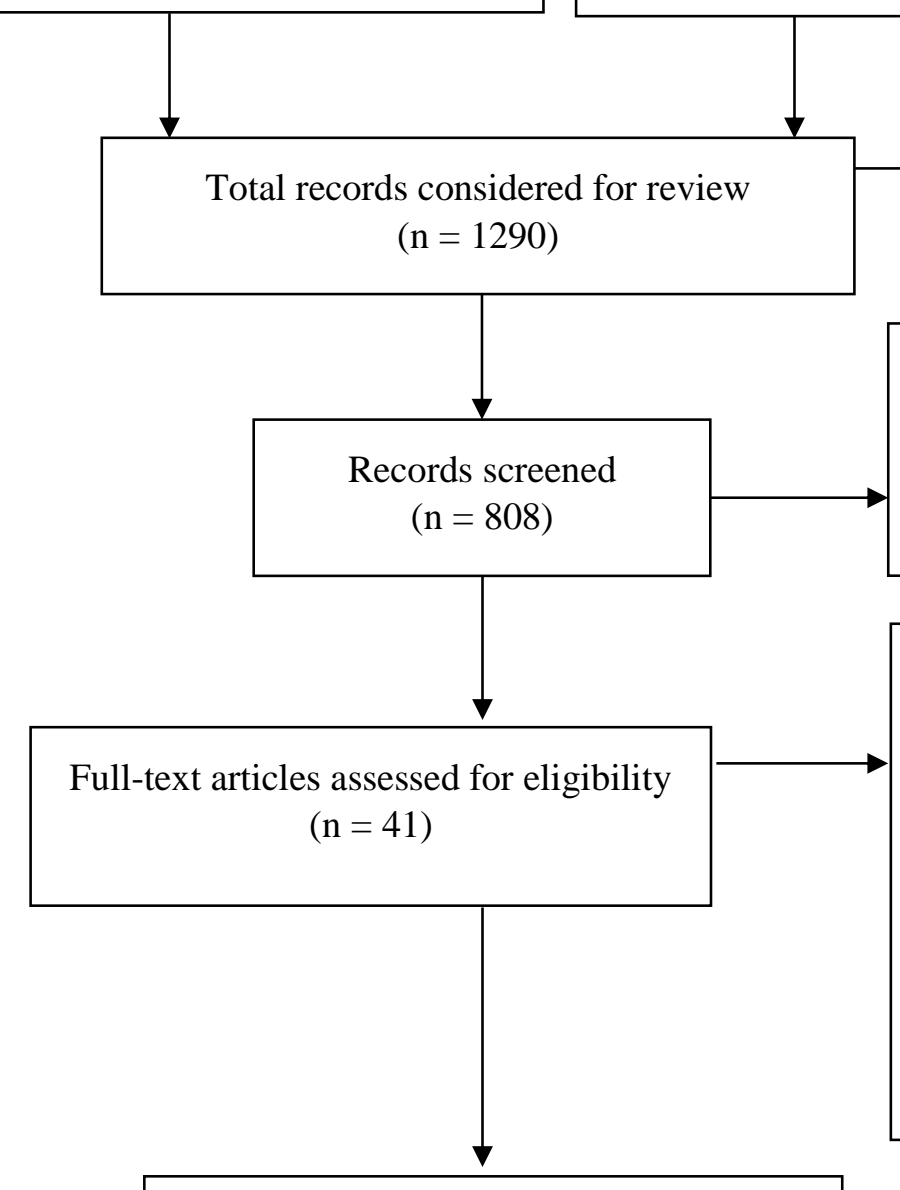

Studies considered for systematic narrative synthesis and meta-analysis

$$
(\mathrm{n}=13)
$$

Additional records identified through other sources

$$
(n=264)
$$

Full-text articles excluded

$$
(\mathrm{n}=28)
$$

Not empirical study in nature

$$
(\mathrm{n}=15)
$$

Not reporting loneliness in quantitative terms $(\mathrm{n}=9)$

Not conducted in Indian population in India $(n=4)$

Figure 1: Flow diagram of the systematic review 


\section{Results}

\section{Study selection}

We found 1026 citations from database searching and 264 citations from additional sources totaling 1290 citations (Figure 1). After removing 482 duplicates, we had 808 citations which were evaluated as per the eligibility criteria and 767 citations were excluded due to non-compliance with the criteria. At this stage, 41 citations were considered for full-text review. After retrieving and evaluating the full texts, 28 articles did not meet our criteria. At the end of the screening process, 13 articles met all the inclusion and exclusion criteria and were recruited in this systematic review (Anil et al., 2016; Bhogle, 1991; Grover et al., 2019, 2018; Malhotra et al., 2019; Mishra et al., 2013; Nayyar and Singh, 2011; Ninan and Gopinath, 2013; Samanta et al., 2012; Shettar et al., 2017; Singh and Mishra, 2009; Susheela et al., 2018; Upmanyu et al., 1994). 
Table 1: Summary of the included studies

\begin{tabular}{|c|c|c|c|c|c|c|}
\hline Source & $\begin{array}{l}\text { Study } \\
\text { location }\end{array}$ & $\begin{array}{l}\text { Study design } \\
\text { and } \\
\text { instruments }\end{array}$ & $\begin{array}{l}\text { Sam } \\
\text { ple } \\
\text { size } \\
\text { (n) }\end{array}$ & $\begin{array}{l}\text { Sample characteristics } \\
\text { and recruitment strategy }\end{array}$ & $\begin{array}{l}\text { Burden of loneliness among the } \\
\text { study participants }\end{array}$ & $\begin{array}{l}\text { Factors, correlates, or conditions } \\
\text { associated with loneliness }\end{array}$ \\
\hline $\begin{array}{l}\text { (Bhogle, } \\
\text { 1991) }\end{array}$ & $\begin{array}{l}\text { Andhra } \\
\text { Pradesh }\end{array}$ & $\begin{array}{l}\text { Cross-sectional } \\
\text { study using } \\
\text { UCLA } \\
\text { Loneliness } \\
\text { Scale }\end{array}$ & 120 & $\begin{array}{l}60 \text { adolescents ( } 30 \text { boys and } \\
30 \text { girls, aged between } 14- \\
15 \text { years) were recruited } \\
\text { from schools; } 60 \text { adults ( } 30 \\
\text { men and } 30 \text { women) were } \\
\text { recruited through } \\
\text { acquaintances; For each age } \\
\text { group, three groups } \\
\text { consisting Hindu, Muslim, } \\
\text { and Christian participants } \\
\text { were recruited }\end{array}$ & $\begin{array}{l}\text { Mean loneliness scores (with SD) } \\
\text { were } 51.3(5.69), 46.1 \text { ( } 5.15) \text {, and } \\
38.7(7.79) \text { among Hindu, Muslim, } \\
\text { and Christian adolescent boys. } \\
\text { Among girls, the scores were } 46.7 \\
(5.88) .42 .7 \text { ( } 7.95), 38 \text { (6.91) } \\
\text { respectively. Mean loneliness } \\
\text { scores were } 41.4 \text { (3.35), } 37.9 \text { (6.7), } \\
\text { 39.55 (10.9) among men and } 39.1 \\
(2.68), 40 \text { (9.13), } 40.6 \text { (10.77) } \\
\text { among women of Hindu, Muslim, } \\
\text { and Christian religions respectively }\end{array}$ & $\begin{array}{l}\text { Religious and cultural influences (Hindu } \\
\text { participants had higher loneliness), age } \\
\text { (older participants experienced less } \\
\text { loneliness), and women (varying levels of } \\
\text { loneliness scores compared to men in } \\
\text { different religious groups) }\end{array}$ \\
\hline $\begin{array}{l}\text { (Upmanyu } \\
\text { et al., } \\
\text { 1994) }\end{array}$ & Punjab & $\begin{array}{l}\text { Cross sectional } \\
\text { study using } \\
\text { UCLA Scale } \\
\text { and Differential } \\
\text { Loneliness } \\
\text { Scale (DLS) }\end{array}$ & 183 & $\begin{array}{l}\text { Male students with a mean } \\
\text { age of } 21.01 \text { years (SD } \\
0.74 \text { ) were recruited from } \\
\text { an University campus }\end{array}$ & $\begin{array}{l}\text { Mean loneliness score was } 38.15 \\
\text { (SD 9.64) among the study } \\
\text { participants with a low of } 22 \text { to a } \\
\text { high of } 72 .\end{array}$ & $\begin{array}{l}\text { Deficiencies in friendship, larger group } \\
\text { relationships, and family relationships had } \\
\text { high correlation ( } \mathrm{r}=0.51 \text { to } 0.69, \mathrm{p}<0.001 \text { ) } \\
\text { with loneliness; deficiencies in romantic or } \\
\text { sexual relationships were also correlated } \\
\text { with loneliness ( } \mathrm{r}=0.19, \mathrm{p}<0.01 \text { ); social } \\
\text { deviance increased loneliness among the } \\
\text { introverts }\end{array}$ \\
\hline $\begin{array}{l}\text { (Singh and } \\
\text { Mishra, } \\
\text { 2009) }\end{array}$ & Delhi & $\begin{array}{l}\text { Cross sectional } \\
\text { study using } \\
\text { revised UCLA } \\
\text { Scale, Beck } \\
\text { depression } \\
\text { inventory, } \\
\text { Sociability } \\
\text { subscale of } \\
\text { Eysenck }\end{array}$ & 55 & $\begin{array}{l}\text { Elderly adult participants } \\
\text { (male }=35, \text { female }=20) \\
\text { mean age } 67 \text { years; } \\
\text { recruited from housing } \\
\text { communities }\end{array}$ & $\begin{array}{l}\text { Mean loneliness score was } 47.43 \\
\text { (SD 7.54) and } 45.75 \text { (SD 9.33) } \\
\text { among male and female participants } \\
\text { respectively }\end{array}$ & $\begin{array}{l}\text { Loneliness was positively correlated with } \\
\text { depression }(\mathrm{p}<0.01) \text { and negatively } \\
\text { correlated with sociability which was not } \\
\text { significant }\end{array}$ \\
\hline
\end{tabular}




\begin{tabular}{|c|c|c|c|c|c|c|}
\hline & & $\begin{array}{l}\text { personality } \\
\text { profiler }\end{array}$ & & & & \\
\hline $\begin{array}{l}\text { (Nayyar } \\
\text { and Singh, } \\
\text { 2011) }\end{array}$ & Punjab & $\begin{array}{l}\text { Cross-sectional } \\
\text { study using } \\
\text { revised UCLA } \\
\text { Scale, revised } \\
\text { Eysenck } \\
\text { Personality } \\
\text { Questionnaire, } \\
\text { Internal- } \\
\text { external scale, } \\
\text { IPAT Self- } \\
\text { Analysis } \\
\text { Anxiety Scale }\end{array}$ & 100 & $\begin{array}{l}\text { Students (male }=50 \text {, } \\
\text { female }=50 \text { ) with mean age } \\
\text { of } 22.2 \text { years (SD } 0.66 \text { ) } \\
\text { were recruited from an } \\
\text { University campus }\end{array}$ & $\begin{array}{l}60 \% \text { participants had shown higher } \\
\text { score on loneliness as per the } \\
\text { UCLA Scale }\end{array}$ & $\begin{array}{l}\text { Loneliness was positively correlated with } \\
\text { neuroticism }(0.37, \mathrm{p}<0.01) \text { expressed as } \\
\text { emotional instability, inadaptability, } \\
\text { depressed mood, week dependable attitudes, } \\
\text { guilt proneness, worry, and lack of } \\
\text { optimism; with psychoticism }(0.18, \text { non- } \\
\text { significant), and anxiety }(0.42, \mathrm{p}<0.01) \text {. } \\
\text { Moreover, loneliness was negatively } \\
\text { correlated with extraversion }(-0.28, \mathrm{p}<0.05) \\
\text { and external locus of control }(-0.23, \mathrm{p}<0.05)\end{array}$ \\
\hline $\begin{array}{l}\text { (Samanta et } \\
\text { al., 2012) }\end{array}$ & $\begin{array}{l}\text { West } \\
\text { Bengal }\end{array}$ & $\begin{array}{l}\text { Cross-sectional } \\
\text { study using } \\
\text { Global School- } \\
\text { based Student } \\
\text { Health Survey } \\
\text { (GSHS) }\end{array}$ & 199 & $\begin{array}{l}\text { Male students (104 urban, } \\
95 \text { rural) were recruited } \\
\text { from schools; mean age was } \\
14.1 \text { (SD 0.87) and } 15.32 \\
\text { (SD 0.96) in urban and rural } \\
\text { participants respectively }\end{array}$ & $\begin{array}{l}\text { The prevalence of self-reported } \\
\text { loneliness was } 17.3 \% \text { and } 9.5 \% \\
\text { among urban and rural participants } \\
\text { respectively }\end{array}$ & $\begin{array}{l}\text { Mental health co-morbidities including } \\
\text { suicidal thoughts (14.1-19.2\%), bullying } \\
(17-46.4 \%) \text {, worry }(10.7-17.2 \%) \text { were } \\
\text { observed among the study participants }\end{array}$ \\
\hline $\begin{array}{l}\text { (Mishra et } \\
\text { al., 2013) }\end{array}$ & $\begin{array}{l}\text { Andhra } \\
\text { Pradesh }\end{array}$ & $\begin{array}{l}\text { Cross-sectional } \\
\text { study using } \\
\text { revised UCLA } \\
\text { Scale and SRQ- } \\
10 \text { (Self- } \\
\text { reporting } \\
\text { Questionnaire) }\end{array}$ & 380 & $\begin{array}{l}\text { People living with HIV } \\
\text { (PLHIV); male } 43.16 \% \text {, } \\
\text { female 56.84\%; } 73.68 \% \\
\text { aged between 20-40 years; } \\
\text { recruited from communities } \\
\text { through ART clinic } \\
\text { registries in two districts }\end{array}$ & $\begin{array}{l}\text { Overall prevalence of loneliness } \\
\text { was } 66.57 \% \text { among the study } \\
\text { participants; Mean loneliness score } \\
\text { was } 51.45 \text { (SD 3.98) and } 53.09 \text { (SD } \\
4.42 \text { ) among male and female } \\
\text { participants }\end{array}$ & $\begin{array}{l}\text { Loneliness was higher among female } \\
\text { participants. Moreover, educational status, } \\
\text { marital status, location of residence, and } \\
\text { opportunistic infections were associated with } \\
\text { loneliness }\end{array}$ \\
\hline $\begin{array}{l}\text { (Ninan and } \\
\text { Gopinath, } \\
\text { 2013) }\end{array}$ & Karnataka & $\begin{array}{l}\text { Cross-sectional } \\
\text { study using } \\
\text { Loneliness and } \\
\text { Social } \\
\text { Dissatisfaction } \\
\text { Questionnaire } \\
\text { and Children's } \\
\text { Attributional }\end{array}$ & 60 & $\begin{array}{l}30 \text { children with locomotor } \\
\text { disabilities and } 30 \text { without } \\
\text { such disabilities; aged } \\
\text { between } 8 \text { to } 13 \text { years } \\
\text { recruited from educational } \\
\text { settings }\end{array}$ & $\begin{array}{l}\text { Mean loneliness score was } 30.267 \\
\text { (SD 9.49) and } 31.77 \text { (SD 7.951) } \\
\text { among children with and without } \\
\text { locomotor disabilities respectively }\end{array}$ & $\begin{array}{l}\text { Loneliness was negatively correlated with } \\
\text { attributional styles both among children with } \\
\text { disabilities }(-0.494, \mathrm{p}<0.006) \text { and those } \\
\text { without disabilities }(-0.4, \mathrm{p}<0.029) \text {; children } \\
\text { with negative and internal-stable-global } \\
\text { attributional styles had higher loneliness and } \\
\text { lower social engagement }\end{array}$ \\
\hline
\end{tabular}




\begin{tabular}{|c|c|c|c|c|c|c|}
\hline & & $\begin{array}{l}\text { Style } \\
\text { Questionnaire- } \\
\text { Revised } \\
\text { (CASQ-R) tools }\end{array}$ & & & & \\
\hline $\begin{array}{l}\text { (Anil et al., } \\
\text { 2016) }\end{array}$ & Karnataka & $\begin{array}{l}\text { Cross-sectional } \\
\text { study using } \\
\text { revised UCLA } \\
\text { Scale }\end{array}$ & 370 & $\begin{array}{l}\text { Elderly adults aged above } \\
60 \text { years; } 39.2 \% \text { males and } \\
60.8 \% \text { females; recruited } \\
\text { from community settings }\end{array}$ & $\begin{array}{l}\text { The prevalence of loneliness was } \\
37.6 \% \text { with a loneliness score of } 50 \\
\text { or above }\end{array}$ & $\begin{array}{l}\text { People with age above } 70 \text { years, smaller } \\
\text { family size ( } 85.7 \% \text {, OR } 8.4 \text { ), separation } \\
\text { from or death of spouse ( } 46.7 \% \text {, OR } 2.1 \text { ), } \\
\text { low number of family members (OR } 8.4 \text { ), } \\
\text { low educational status (OR } 2.3 \text { ), functional } \\
\text { disabilities (upto } 61 \% \text {, OR 3.2), financial } \\
\text { dependency ( } 38.5 \% \text { ) and presence of co- } \\
\text { morbid noncommunicable diseases ( } 37.7 \% \text { ) } \\
\text { had high loneliness }\end{array}$ \\
\hline $\begin{array}{l}\text { (Shettar et } \\
\text { al., 2017) }\end{array}$ & Karnataka & $\begin{array}{l}\text { Cross-sectional } \\
\text { study using } \\
\text { revised UCLA } \\
\text { Scale, Bergen } \\
\text { Facebook } \\
\text { Addiction Scale } \\
\text { (BFAS) }\end{array}$ & 100 & $\begin{array}{l}\text { Post-graduate students with } \\
\text { mean age of } 27.55 \text { (SD } \\
\text { 2.88) years; } 54 \% \text { male, } 46 \% \\
\text { female; recruited from } \\
\text { academic setting }\end{array}$ & $\begin{array}{l}\text { Mean loneliness score was } 51.66 \\
\text { (SD 6.95), 53.03 (SD 5.51), and } \\
55.85 \text { (SD 5.63) among students } \\
\text { with no, suspected, and identified } \\
\text { Facebook addiction respectively }\end{array}$ & $\begin{array}{l}\text { Severity of loneliness was correlated }(0.239, \\
\mathrm{p}<0.05) \text { with Facebook addiction }\end{array}$ \\
\hline $\begin{array}{l}\text { (Susheela } \\
\text { et al., } \\
2018)\end{array}$ & Karnataka & $\begin{array}{l}\text { Cross-sectional } \\
\text { study using } \\
\text { revised UCLA } \\
\text { Scale, Geriatric } \\
\text { Depression } \\
\text { Scale, Activities } \\
\text { of Daily Living } \\
\text { Scale for } \\
\text { elderly }\end{array}$ & 600 & $\begin{array}{l}\text { Elderly adults aged above } \\
60 \text { years; } 42.2 \% \text { males and } \\
57.8 \% \text { females; recruited } \\
\text { from rural areas }\end{array}$ & $\begin{array}{l}\text { The overall prevalence of loneliness } \\
\text { was } 4.8 \% \text { ( } 3.8 \% \text { low, } 1 \% \text { moderate } \\
\text { to high) }\end{array}$ & $\begin{array}{l}\text { Marital status, education attainment, } \\
\text { financial status, family structure, and } \\
\text { presence of co-morbid conditions were } \\
\text { associated with loneliness; the study } \\
\text { population had a high prevalence }(40.16 \%) \\
\text { of depression }\end{array}$ \\
\hline $\begin{array}{l}\text { (Grover et } \\
\text { al., 2018) }\end{array}$ & $\begin{array}{l}\text { Multicentr } \\
\text { ic study }\end{array}$ & $\begin{array}{l}\text { Cross-sectional } \\
\text { study using } \\
\text { Geriatric } \\
\text { Depression } \\
\text { Rating Scale } \\
\text { (GDS-30), }\end{array}$ & 488 & $\begin{array}{l}\text { Elderly adults aged above } \\
60 \text { years; Mean age } 66.55 \\
\text { (SD 5.83) years; } 53.7 \% \\
\text { male, } 46.3 \% \text { female; } \\
\text { recruited from eight study } \\
\text { centers }\end{array}$ & $\begin{array}{l}\text { The prevalence of loneliness was } \\
77.3 \% \text { with mean loneliness score } \\
\text { of } 27.93 \text { (SD 13.96); } 62.5 \% \text { of the } \\
\text { participants reported lack of } \\
\text { companionship, } 58.7 \%\end{array}$ & $\begin{array}{l}\text { Relationship status (being single), older age, } \\
\text { low education, presence of family history of } \\
\text { mental illness, presence of comorbid } \\
\text { physical and mental illnesses (depression } \\
\text { and anxiety), and absence of substance } \\
\text { abuse were associated with higher loneliness }\end{array}$ \\
\hline
\end{tabular}




\begin{tabular}{|c|c|c|c|c|c|c|}
\hline & & $\begin{array}{l}\text { Generalized } \\
\text { Anxiety } \\
\text { disorder } \\
\text { questionnaire } \\
\text { (GAD-7), } \\
\text { Columbia } \\
\text { Suicide } \\
\text { Severity Rating } \\
\text { Scale } \\
\text { (C-SSRS), } \\
\text { UCLA Scale, } \\
\text { Patient Health } \\
\text { Questionnaire } \\
\text { (PHQ-15), } \\
\text { and Revised } \\
\text { Social } \\
\text { Connectedness } \\
\text { Scale }\end{array}$ & & & $\begin{array}{l}\text { reported being left out in life, and } \\
56.5 \% \text { reported felt isolated from } \\
\text { others }\end{array}$ & \\
\hline $\begin{array}{l}\text { (Grover et } \\
\text { al., 2019) }\end{array}$ & Punjab & $\begin{array}{l}\text { Cross-sectional } \\
\text { study using } \\
\text { revised UCLA } \\
\text { Scale, Geriatric } \\
\text { Depression } \\
\text { Scale, GAD-7 } \\
\text { Scale, } \\
\text { Vulnerability } \\
\text { to Abuse } \\
\text { Screening Scale }\end{array}$ & 296 & $\begin{array}{l}\text { Elderly adults aged above } \\
60 \text { years; } 45.6 \% \text { male, } \\
54.4 \% \text { female; recruited } \\
\text { from rural community } \\
\text { clinics }\end{array}$ & $\begin{array}{l}\text { Overall prevalence of loneliness } \\
\text { was } 55.4 \% ; 50.7 \%, 13.2 \%, 17.6 \% \text {, } \\
\text { and } 18.6 \% \text { participants experienced } \\
\text { low, moderate, moderately high, } \\
\text { and high level of loneliness }\end{array}$ & $\begin{array}{l}\text { Loneliness was associated with living with } \\
\text { joint families, presence of hypertension, } \\
\text { diabetes, anxiety, and substance abuse }\end{array}$ \\
\hline $\begin{array}{l}\text { (Malhotra } \\
\text { et al., } \\
\text { 2019) }\end{array}$ & Haryana & $\begin{array}{l}\text { Cross sectional } \\
\text { study using } \\
\text { semi-structured } \\
\text { questionnaire }\end{array}$ & 836 & $\begin{array}{l}\text { Young individuals with } \\
\text { mean age of } 20.6 \text { (SD 1.9) } \\
\text { years and } 11.8 \text { (SD 2.8) } \\
\text { mean years of schooling }\end{array}$ & $\begin{array}{l}\text { Age adjusted prevalence of } \\
\text { loneliness was } 28.6 \% \text { (95\% CI } \\
28.35-28.83)\end{array}$ & $\begin{array}{l}\text { Loneliness was associated with current } \\
\text { substance abuse, premarital sexual } \\
\text { intercourse, and serious injury }\end{array}$ \\
\hline
\end{tabular}




\section{Study characteristics and instruments}

Among the included studies, only three were published before 2010 whereas most $(n=10)$ of the studies were published after 2010 (see Table 1). Studies were conducted in different states of India. Most $(n=4)$ studies were conducted in Karnataka (Anil et al., 2016; Ninan and Gopinath, 2013; Shettar et al., 2017; Susheela et al., 2018), followed by Punjab ( $n=3$ ) (Grover et al., 2019; Nayyar and Singh, 2011; Upmanyu et al., 1994), Andhra Pradesh ( $\mathrm{n}=2$ ) (Bhogle, 1991; Mishra et al., 2013), Delhi ( $\mathrm{n}=1)$ (Singh and Mishra, 2009), Haryana ( $=1)$ (Malhotra et al., 2019), and West Bengal ( $\mathrm{n}=1$ ) (Samanta et al., 2012). A study by Grover et al. included participants from multiple states of India (Grover et al., 2018). In most of the studies ( $\mathrm{n}=10)$, University of California Los Angeles (UCLA) Loneliness Scale was used to evaluate loneliness among the participants (Anil et al., 2016; Bhogle, 1991; Grover et al., 2019, 2018; Mishra et al., 2013; Nayyar and Singh, 2011; Shettar et al., 2017; Singh and Mishra, 2009; Susheela et al., 2018; Upmanyu et al., 1994). Other scales used for assessing loneliness, associated sociodemographic, and clinical conditions included differential loneliness scale (Upmanyu et al., 1994), Eysenck personality profiler (Singh and Mishra, 2009), loneliness and social dissatisfaction questionnaire (Ninan and Gopinath, 2013), Columbia suicide severity scale (Grover et al., 2018), patient health questionnaire (Grover et al., 2018), social connectedness scale (Grover et al., 2018), generalized anxiety disorder questionnaire (Grover et al., 2018), vulnerability to abuse screening scale (Grover et al., 2019), Bergan Facebook addiction scale (Shettar et al., 2017), Beck depression inventory (Singh and Mishra, 2009), internal-external scale (Nayyar and Singh, 2011), Institute for Personality and Ability Testing (IPAT) self-analysis anxiety scale (Nayyar and Singh, 2011), global school student health survey (Samanta et al., 2012), children attribution style questionnaire (Ninan and Gopinath, 2013), geriatric depression scale (Grover et al., 2019, 2018), activities of daily living scale (Susheela et al., 2018), and self-reported questionnaire (Malhotra et al., 2019; Mishra et al., 2013).

\section{Sample characteristics}

Thirteen studies in this review represent a total of 3787 participants, while individual studies had a varying number of participants ranging from 55 to 836. Six studies recruited students from different academic institutions (Bhogle, 1991; Nayyar and Singh, 2011; Ninan and Gopinath, 2013; Samanta et al., 2012; Shettar et al., 2017; Upmanyu et al., 1994). For example, Shettar et al. 
recruited post-graduate students with a mean age of 27.55 years (SD 2.88) from academic setting. Moreover, five studies recruited elderly participants mostly from community settings (Anil et al., 2016; Grover et al., 2019, 2018; Singh and Mishra, 2009; Susheela et al., 2018). In a study by Bhogle S., both children and adult participants were recruited to evaluate how they perceived loneliness (Bhogle, 1991). A few studies included participants with preexisting chronic conditions (Mishra et al., 2013; Ninan and Gopinath, 2013). For example, Ninan et al. assessed loneliness among children with locomotor disabilities (Ninan and Gopinath, 2013). Another study by Mishra et al. examined loneliness among people living with HIV (PLHIV) (Mishra et al., 2013). While most of the studies were conducted in urban areas, Samanta et al. recruited two groups of participants from urban and rural areas (Samanta et al., 2012).

\section{Quality assessment of the recruited studies}

Quality assessment using the NHLBI tool revealed internal validity and risk of bias for each study (see Supplementary file). Moreover, this tool was used to rate the overall quality of the included studies. Among 13 studies included in this review, five were rated as good (Anil et al., 2016; Grover et al., 2019; Malhotra et al., 2019; Mishra et al., 2013; Susheela et al., 2018), seven studies as fair (Bhogle, 1991; Grover et al., 2018; Nayyar and Singh, 2011; Ninan and Gopinath, 2013; Samanta et al., 2012; Shettar et al., 2017; Upmanyu et al., 1994), and one study was rated as poor (Singh and Mishra, 2009) following the quality evaluation using NHLBI tool.

\section{Severity of loneliness in India}

Among 13 studies included in this review, nine studies reported the severity of loneliness as mean loneliness scores ranging between 27.93 (SD 13.96) and 55.85 (SD 5.63) (Anil et al., 2016; Bhogle, 1991; Grover et al., 2018; Mishra et al., 2013; Nayyar and Singh, 2011; Ninan and Gopinath, 2013; Shettar et al., 2017; Singh and Mishra, 2009; Upmanyu et al., 1994). Three studies reported both loneliness scores and prevalence of loneliness among the study participants (Anil et al., 2016; Grover et al., 2018; Mishra et al., 2013). Seven studies reported prevalence of loneliness among study participants (Anil et al., 2016; Grover et al., 2019, 2018; Malhotra et al., 2019; Mishra et al., 2013; Samanta et al., 2012; Susheela et al., 2018), which were included in the meta-analysis 
(Figure 2). The pooled prevalence of loneliness was $41 \%$ (95\% confidence interval [CI]: $18 \%$ $63 \%, I^{2}=99.66 \%, p=0.00$ ) among 3169 participants representing the seven prevalence studies. Moreover, five studies reported the prevalence using the UCLA scale, which revealed a pooled prevalence of loneliness as 48\% (95\% CI: 14\%-83\%, $\left.I^{2}=99.77 \%, p=0.00\right)$ among 2134 eligible participants (Anil et al., 2016; Grover et al., 2019, 2018; Mishra et al., 2013; Susheela et al., 2018). Furthermore, two studies reported prevalence among children, adolescent, and young adults $(\mathrm{n}=$ 1035) (Malhotra et al., 2019; Samanta et al., 2012), which had a pooled prevalence of $24 \%$ (95\% CI: $22 \%-27 \%$ ). In addition, four studies reported the prevalence of loneliness among elderly adults $(n=1754)$ (Anil et al., 2016; Grover et al., 2019, 2018; Susheela et al., 2018), which revealed a pooled prevalence of $44 \%$ (95\% CI: 5\%-83\%) All the findings in the meta-analysis had shown significant heterogeneity $\left(I^{2}=99.8 \%, p=0.00\right)$.

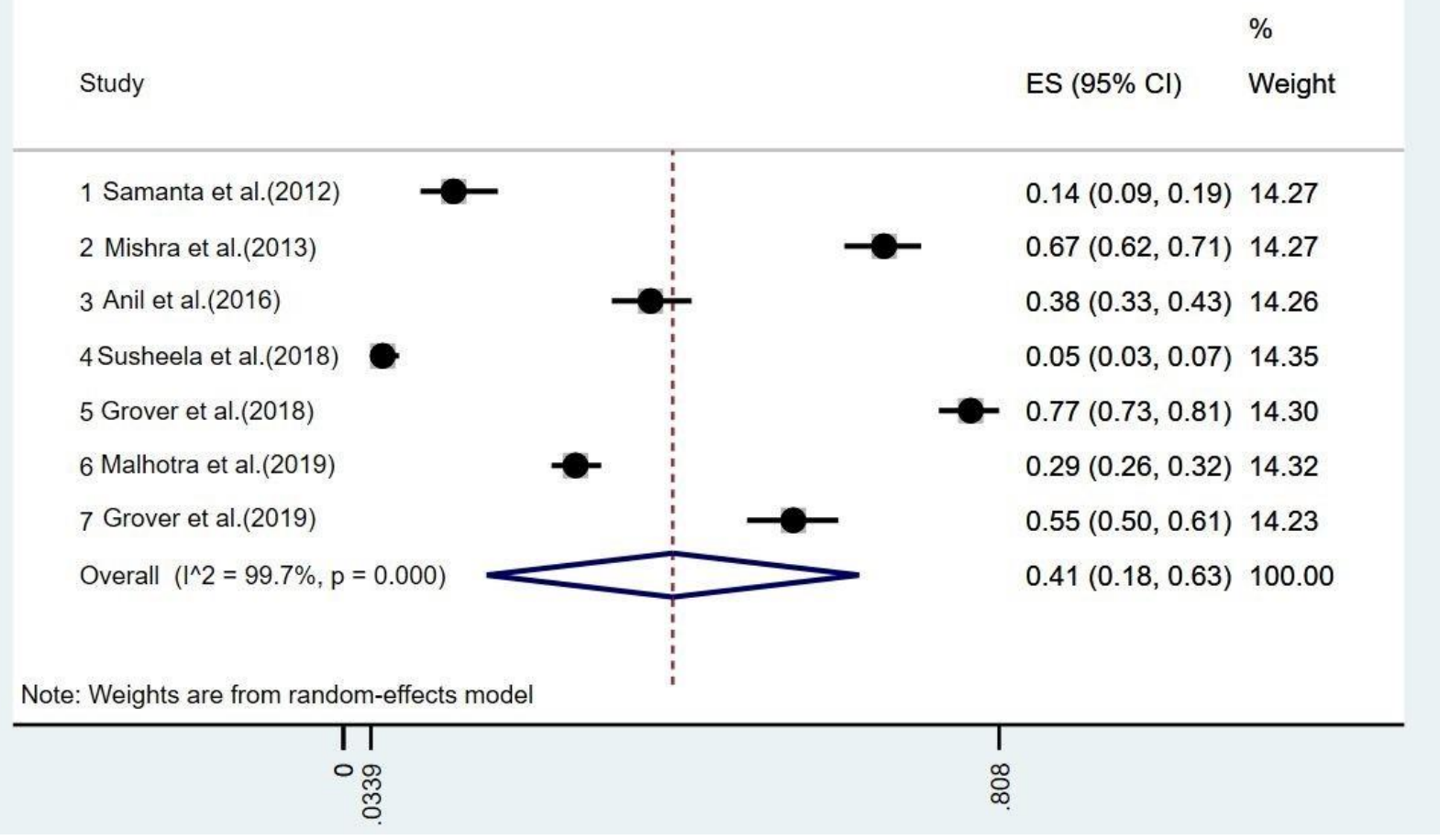

Figure 2: The pooled prevalence of loneliness in India

Factors associated with loneliness in India 
Studies included in this review reported several sociodemographic factors associated with loneliness. Age of the participants was a common factor associated with loneliness. For example, older age was associated with loneliness in studies conducted by Anil et al. and Grover et al. (Anil et al., 2016; Grover et al., 2018), whereas Bhogle found younger participants as more lonely (Bhogle, 1991). Moreover, family structure and marital status were associated with loneliness. For example, a study by Anil et al. found older adults living without a spouse were 1.9 times more likely to experience loneliness (Anil et al., 2016). Financial status was associated with loneliness across studies in this review. Another study by Susheela et al. found a significant association $(p$ $<0.001$ ) of loneliness with family income and source of income (Susheela et al., 2018). Furthermore, several individual characteristics of the participants were associated with loneliness, which included extraversion, external locus of control, and attributional style (Nayyar and Singh, 2011; Ninan and Gopinath, 2013). Other sociodemographic factors associated with loneliness included religious practices, gender, group membership, sociability, educational attainment, and lack of recreational activities (Bhogle, 1991; Grover et al., 2018; Susheela et al., 2018; Upmanyu et al., 1994).

Studies also reported several clinical conditions associated with loneliness. Preexisting noncommunicable diseases were associated with loneliness. For example, a study by Grover et al. reported people with generalized anxiety disorder (OR 2.07, $\mathrm{p}<0.036$ ) and diabetes mellitus (OR 2.52, $\mathrm{p}<0.014$ ) had a higher likelihood of experiencing loneliness (Grover et al., 2019, 2018). Moreover, the functional abilities of the participants were associated with loneliness. Anil et al. found basic activity disability as a risk factor associated with loneliness (OR 3.2, p <0.05) (Anil et al., 2016). Other clinical conditions associated with loneliness included depression, neuroticism, psychoticism, suicidal thoughts, bullying, worrying, presence of opportunistic infections, family history of mental illness, serious injury, addiction to social media, and substance abuse (Grover et al., 2019, 2018; Malhotra et al., 2019; Nayyar and Singh, 2011; Shettar et al., 2017; Susheela et al., 2018).

\section{Discussion}


This systematic review included 13 studies on diverse populations. Of the 13, two were on adolescents, four were on young people, one was on adults-which also included adolescents, five were on only elderly adults and two were on special groups like PLHIV and children with disability. The number of studies considered for review is less in number as they are the only studies conducted in India over a period of 1991 to 2019. Notably, the reviewed studies attempted to understand loneliness along the spectrum with respect to different age groups and also diverse possible correlates. The exploration and review of the studies helped in assessing not only the prevalence of loneliness among different population groups but also assisted in identifying the common correlates for loneliness across all population groups studied.

This review also found that it is widely prevalent not only among the elderly individuals but also in the young population. The study by Bhogle has shown that a greater proportion of adolescents scored on loneliness compared to adult males and females (Bhogle, 1991). Similarly, another study reported the prevalence of self-reported loneliness as $17.3 \%$ and $9.5 \%$ among urban and rural adolescents respectively (Samanta et al., 2012). In another study, of the sampled young people with an average age of 22 years, 60\% reported higher scores on loneliness (Nayyar and Singh, 2011). This indicates how the transition period of adolescence turns into a period of a quandary, when the complex psychosomatic and social dynamics among the adolescents may result in social isolation and loneliness. Nonetheless, this review found that children in the age group of 8-13 years-both with and without disabilities have reported loneliness. This is a serious mental health concern for India, which already has a high burden of mental disorders among children and adolescents (Hossain and Purohit, 2019). Moreover, among special sub-groups like PLHIV, the prevalence of loneliness was reported among around $66 \%$ of the studied population, which was associated with the educational status, marital status, residence and opportunistic infection (Mishra et al., 2013). While the importance of social support cannot be undermined at any of the stages of life, it attains crucial importance during the old age. The studies have shown loneliness and depression to be positively correlated and negative correlated with sociability (Singh and Mishra, 2009). The perception of loneliness is also reflective of the culture, and religion might also play a role in it (Rote et al., 2013). Religion determines the customs to a great extent and custom determines the daily life. For both the adolescent group and adult population, Hindus have scored more on loneliness compared to those professing Islam and Christianity. While the proportion 
remained in the same order for adolescents and adults belonging to Hinduism and Islam, it showed an increase for both men and women belonging to Christianity compared to adolescents of the same religion (Bhogle, 1991). Furthermore, the high burden of loneliness can be attributable due to a decline in stable close relationships as well as overall social capital (Böger and Huxhold, 2018). These are the times when a collectivist society like India is moving towards individualism, and as societies make this transition, the stress shifts to independence rather than inter-dependence, which ultimately becomes a cause of loneliness (Bhogle, 1991). Older adults living with noncommunicable diseases are at a higher risk for loneliness (Grover et al., 2019). It is suggested that mutual help groups kind of models can be developed to help in "re-peopling" elderly (Grover et al., 2019). Primary healthcare centers should help in improving the overall health and morale amongst the elderly by stamping down their apprehensions and anxieties. It is, therefore, very important that loneliness and social isolation are taken into consideration when mental health issues are identified or reported by people.

Similar to other life situations and age groups, loneliness among PLHIV also has been found to be affected by strong networking among PLHIV, quality counseling, and NGO support. Psychological research with PLHIV has reflected a conceptual shift towards perceiving HIV and AIDS as a chronic disease (Chander et al., 2006), therefore, buffers which had shown to be working against loneliness for PLHIV could be thought for other people also. Comparison of loneliness between pre-antiretroviral therapy (ART) and on ART showed that, among those PLHIV who were on pre-ART, the loneliness was less, and depression was found to be more (Chander et al., 2006). This may be due to the fact that once they are registered for ART they attend the "community care centers" where they interact more with other PLHIV who are on ART, NGOs, and counselors. Other studies with the general population have also emphasized the role of social isolation in loneliness and a significant relationship between depression and loneliness (Yanguas et al., 2018); hence, effort needs to be channelized to establish and maintain contacts on a regular basis. Barriers to participation in socialization activities need to be considered, including practice and functional barriers, as well as perceived social barriers to becoming involved in groups. One of the indicators of changing times is the use of internet and this too has contributed to loneliness in the garb of connectedness. Young individuals who use the internet excessively spend less time in face to face interaction which may result in loneliness, depression, low self-esteem and anxiety (Ybarra et al., 
2005). Also, sociocultural values in joint or extended families, which used to offer assurance against loneliness of the people in the family, may have changed over time affecting psychosocial health at population level. Research findings suggest that loneliness in joint family needs to be considered from the perspective of changing norms whereby despite the presence of many people in the same household, the interaction is reduced due to the increasing use of gadgets (Drago, 2015). Such social and ecological changes should be evaluated carefully to better understand the roots of loneliness in the context of India. Currently, loneliness is not being recognized or assessed as a mental health problem in India. The National Mental Health Survey of India (2015-16) reported varying burden of mental health issues with little emphasis on loneliness (Murthy, 2017). In addition, South Asian countries including India have a high prevalence of mental disorders (Hossain et al., 2020b), where loneliness may impose further psychosocial burden among the affected individuals. Loneliness should be studied in the context of other mental disorders, especially among vulnerable individuals who have multiple coexisting mental disorders (Hossain et al., 2020a). Further research is required in this area to estimate and highlight prevalence among different population and sub population groups, their experience of loneliness, psychiatric comorbidities, and different risk as well protective factors associated with loneliness.

Loneliness could be tackled by multi-pronged approaches. Psychosocial interventions along with clinical measures are required to prevent predisposing factors associated with loneliness, depression, suicidal attempts, and other closely associated mental health problems. A metaanalysis evaluated 50 intervention studies comprising 12 pre-post studies, 18 non-randomized comparison studies, and 20 randomized studies highlighting the effectiveness of different interventions to reduce loneliness among the study participants (Masi et al., 2011). Strategies to address loneliness include improving social skills, increasing psychosocial support, enhancing opportunities to improve social networks and connections, and addressing maladaptive social cognition (Cacioppo et al., 2015). However, there is a lack of evidence on the application of such measures in the context of India. Future research and development should leverage the lessons from different contexts to address the loneliness epidemic in India. Moreover, loneliness coexists with noncommunicable morbidities, especially among the elderly. India has a high prevalence of a wide range of noncommunicable diseases, which makes it essential to explore and address loneliness to alleviate the potential public health burden and health disparities in India. It is critical 
to improve the awareness of the healthcare professionals, researchers, and policymakers through capacity building exercises, which may help in identifying loneliness as a co-factor of many of the seemingly age-related health problems. Collaboration between researchers and community practitioners, institutions, and other stakeholders is essential to develop effective mechanisms, both for research and practice, to address the growing problem of loneliness in India.

This review has several limitations. Firstly, there is a possibility that we could not review the studies indexed in databases that we did not search. Secondly, we excluded dissertations and opinions, which could have provided citations or references to studies that we could not find through our systematic search process. Thirdly, the data extracted from the recruited studies were not homogenous to conduct quantifiable evaluation of the risk factors associated with loneliness. Fourthly, most studies were conducted among vulnerable populations, therefore, the findings of this review may not be generalized. Lastly, marked heterogeneity in terms of sociodemographic and psychosocial measurements did not allow to evaluate the moderating effects of those constructs, which remain a limitation of this review. However, we conducted this review involving at least two reviewers at each step of systematic analysis to reduce biases and attempted to synthesize the current evidence from empirical studies. To our best knowledge, this is the first systematic review reporting the status and correlates of loneliness in India. Future studies should be conducted addressing these limitations to better understand the psychosocial epidemiology of loneliness in India.

\section{Conclusion}

Despite a low number of studies and marked heterogeneity within the same, this review provides a ground to recognize loneliness as a mental health problem in India. Moreover, the findings of this review suggest a high epidemiological and psychosocial burden of loneliness and different correlates, which necessitates preventive and therapeutic interventions at different socioecological levels. However, future research with large sample, covering diverse geographical areas and age groups, and longitudinal designs will be better able to provide substantial evidence to the systems accountable for the health and well-being of its people to conceptualize and design measures to 
address loneliness. Both intrinsic and extrinsic factors need to be identified for tackling lonelinessas this will go a long way to curb the menace of the increasing burden of mental illness in India.

Declaration of interests: We declare no conflicts of interest.

Acknowledgment: None.

Role of the funding source: No funding was received at any stage of conducting this systematic review or preparing this manuscript. 


\section{References}

Alexandra, P., Angela, H., Ali, A., 2018. Loneliness in people with intellectual and developmental disorders across the lifespan: A systematic review of prevalence and interventions. J. Appl. Res. Intellect. Disabil. 31, 643-658. https://doi.org/10.1111/jar.12432

Anil, R., Prasad, K., Puttaswamy, M., 2016. The prevalence of loneliness and its determinants among geriatric population in Bengaluru City, Karnataka, India. Int. J. Community Med. Public Heal. 3, 3246-3251. https://doi.org/10.18203/2394-6040.ijcmph20163944

Bandari, R., Khankeh, H.R., Shahboulaghi, F.M., Ebadi, A., Keshtkar, A.A., Montazeri, A., 2019. Defining loneliness in older adults: protocol for a systematic review. Syst. Rev. 8, 26. https://doi.org/10.1186/s13643-018-0935-y

Beutel, M.E., Klein, E.M., Brähler, E., Reiner, I., Jünger, C., Michal, M., Wiltink, J., Wild, P.S., Münzel, T., Lackner, K.J., Tibubos, A.N., 2017. Loneliness in the general population: prevalence, determinants and relations to mental health. BMC Psychiatry 17, 97. https://doi.org/10.1186/s12888-017-1262-X

Bhogle, S., 1991. Perception of loneliness as an index of culture and age. Psychol. Stud. (Mysore). 36, 174-179.

Böger, A., Huxhold, O., 2018. Age-related changes in emotional qualities of the social network from middle adulthood into old age: How do they relate to the experience of loneliness? Psychol. Aging 33, 482-496. https://doi.org/10.1037/pag0000222

Cacioppo, J.T., Cacioppo, S., 2018. The growing problem of loneliness. Lancet (London, England) 391, 426. https://doi.org/10.1016/S0140-6736(18)30142-9

Cacioppo, S., Grippo, A.J., London, S., Goossens, L., Cacioppo, J.T., 2015. Loneliness: clinical import and interventions. Perspect. Psychol. Sci. 10, 238-49. https://doi.org/10.1177/1745691615570616

Chander, G., Himelhoch, S., Moore, R.D., 2006. Substance Abuse and Psychiatric Disorders in HIV-Positive Patients. Drugs 66, 769-789. https://doi.org/10.2165/00003495-20066606000004 
De Jong Gierveld, J., Van der Pas, S., Keating, N., 2015. Loneliness of Older Immigrant Groups in Canada: Effects of Ethnic-Cultural Background. J. Cross. Cult. Gerontol. 30, 251-268. https://doi.org/10.1007/s10823-015-9265-X

DerSimonian, R., Laird, N., 1986. Meta-analysis in clinical trials. Control. Clin. Trials 7, 177188. https://doi.org/10.1016/0197-2456(86)90046-2

Drago, E., 2015. The Effect of Technology on Face-to-Face Communication. Elon J. Undergrad. Res. Commun. 6, 13-19.

Grover, S., 2019. Loneliness: Does it need attention! J. Geriatr. Ment. Heal. 6, 1. https://doi.org/10.4103/jgmh.jgmh_27_19

Grover, S., Avasthi, A., Sahoo, S., Lakdawala, B., Dan, A., Nebhinani, N., Dutt, A., Tiwari, S.C., Gania, A.M., Subramanyam, A.A., Kedare, J., Suthar, N., 2018. Relationship of loneliness and social connectedness with depression in elderly: A multicentric study under the aegis of Indian Association for Geriatric Mental Health. J Geriatr Ment Heal. 5, 99-106. https://doi.org/10.4103/jgmh.jgmh

Grover, S., Verma, M., Singh, T., Dahiya, N., Nehra, R., 2019. Loneliness and its correlates amongst elderly attending non-communicable disease rural clinic attached to a tertiary care centre of North India. Asian J. Psychiatr. 43, 189-196. https://doi.org/10.1016/j.ajp.2019.06.001

Hawkley, L.C., Cacioppo, J.T., 2010. Loneliness matters: a theoretical and empirical review of consequences and mechanisms. Ann. Behav. Med. 40, 218-27. https://doi.org/10.1007/s12160-010-9210-8

Holt-Lunstad, J., Smith, T.B., Baker, M., Harris, T., Stephenson, D., 2015. Loneliness and Social Isolation as Risk Factors for Mortality. Perspect. Psychol. Sci. 10, 227-237. https://doi.org/10.1177/1745691614568352

Holt-Lunstad, J., Smith, T.B., Layton, J.B., 2010. Social Relationships and Mortality Risk: A Meta-analytic Review. PLoS Med. 7, e1000316. https://doi.org/10.1371/journal.pmed.1000316

Hossain, M.M., Khan, N., Sultana, A., Ma, P., McKyer, E.L.J., Ahmed, H.U., Purohit, N., 2020a. 
Prevalence of comorbid psychiatric disorders among people with autism spectrum disorder:

An umbrella review of systematic reviews and meta-analyses. Psychiatry Res. 112922. https://doi.org/10.1016/j.psychres.2020.112922

Hossain, M.M., Purohit, N., 2019. Improving child and adolescent mental health in India: Status, services, policies, and way forward. Indian J. Psychiatry. https://doi.org/10.4103/psychiatry.IndianJPsychiatry_217_18

Hossain, M.M., Purohit, N., Sultana, A., Ma, P., J McKyer, E.L., Uddin Ahmed, H., $2020 b$. Prevalence of mental disorders in South Asia: an umbrella review of systematic reviews and meta-analyses. Asian J. Psychiatr. 102041. https://doi.org/10.1016/j.ajp.2020.102041

Jeyalakshmi, S., Chakrabarti, S., Gupta, N., 2011. Situation analysis of the elderly in India.

Lee, E.E., Depp, C., Palmer, B.W., Glorioso, D., Daly, R., Liu, J., Tu, X.M., Kim, H.-C., Tarr, P., Yamada, Y., Jeste, D. V., 2018. High prevalence and adverse health effects of loneliness in community-dwelling adults across the lifespan: role of wisdom as a protective factor. Int. Psychogeriatrics 1-16. https://doi.org/10.1017/S1041610218002120

Levy, I., Attias, S., Ben-Arye, E., Bloch, B., Schiff, E., 2017. Complementary medicine for treatment of agitation and delirium in older persons: A systematic review and narrative synthesis. Int. J. Geriatr. Psychiatry 32, 492-508. https://doi.org/10.1002/gps.4685

Liberati, A., Altman, D.G., Tetzlaff, J., Mulrow, C., Gotzsche, P.C., Ioannidis, J.P., Clarke, M., Devereaux, P.J., Kleijnen, J., Moher, D., 2009. The PRISMA statement for reporting systematic reviews and meta-analyses of studies that evaluate healthcare interventions: explanation and elaboration. Bmj 339, b2700. https://doi.org/10.1136/bmj.b2700

Malhotra, S., Kant, S., Ahamed, F., Rath, R., Kaladivani, M., Gupta, S.K., Ramadass, S., Pathak, V.K., Jaiswal, A., Parthasarathy, R., Acharya, B.P., Dwarakanathan, V., 2019. Health behaviors, outcomes and their relationships among young men aged 18-24 years in a rural area of north India: A cross-sectional study. PLoS One 14, e0221493. https://doi.org/10.1371/journal.pone.0221493

Masi, C.M., Chen, H.-Y., Hawkley, L.C., Cacioppo, J.T., 2011. A meta-analysis of interventions to reduce loneliness. Pers. Soc. Psychol. Rev. 15, 219-66. 
https://doi.org/10.1177/1088868310377394

Mishra, S.K., Behera, U.K., Jena, S.K., 2013. Assessment and evaluation of depression and loneliness among people living with HIV in selected places of coastal Andhra Pradesh. Indian J. Public Heal. Res. Dev. 4, 261-266. https://doi.org/10.5958/j.0976-5506.4.3.121

Mullen, R.A., Tong, S., Sabo, R.T., Liaw, W.R., Marshall, J., Nease, D.E., Krist, A.H., Frey, J.J., 2019. Loneliness in Primary Care Patients: A Prevalence Study. Ann. Fam. Med. 17, 108115. https://doi.org/10.1370/afm.2358

Murthy, R.S., 2017. National Mental Health Survey of India 2015-2016. Indian J. Psychiatry 59, 21-26. https://doi.org/10.4103/psychiatry.IndianJPsychiatry_102_17

Mushtaq, R., Shoib, S., Shah, T., Mushtaq, S., 2014. Relationship between loneliness, psychiatric disorders and physical health? A review on the psychological aspects of loneliness. J. Clin. Diagn. Res. 8, WE01-4. https://doi.org/10.7860/JCDR/2014/10077.4828

National Heart Lung and Blood Institute (NHLBI), 2018. Quality Assessment Tool for Observational Cohort and Cross-sectional Studies [WWW Document]. URL https://www.nhlbi.nih.gov/health-topics/study-quality-assessment-tools (accessed 8.25.19).

Nayyar, S., Singh, B., 2011. Personality correlates of loneliness. J. Indian Acad. Appl. Psychol. $37,163-168$.

Nethan, S., Sinha, D., Mehrotra, R., 2017. Non Communicable Disease Risk Factors and their Trends in India. Asian Pac. J. Cancer Prev. 18, 2005-2010. https://doi.org/10.22034/APJCP.2017.18.7.2005

Ninan, P.A., Gopinath, J.K., 2013. Exploring loneliness in relation to attributional styles in children with locomotor disabilities. J. Indian Acad. Appl. Psychol. 39, 237-244.

Rafnsson, S.B., Orrell, M., d'Orsi, E., Hogervorst, E., Steptoe, A., 2017. Loneliness, Social Integration, and Incident Dementia Over 6 Years: Prospective Findings From the English Longitudinal Study of Ageing. Journals Gerontol. Ser. B. https://doi.org/10.1093/geronb/gbx087

Rote, S., Hill, T.D., Ellison, C.G., 2013. Religious attendance and loneliness in later life. 
Gerontologist 53, 39-50. https://doi.org/10.1093/geront/gns063

Rubin, R., 2017. Loneliness Might Be a Killer, but What's the Best Way to Protect Against It? JAMA 318, 1853. https://doi.org/10.1001/jama.2017.14591

Samanta, A., Mukherjee, S., Ghosh, S., Dasgupta, A., 2012. Mental health, protective factors and violence among male adolescents: a comparison between urban and rural school students in West Bengal. Indian J. Public Health 56, 155-158. https://doi.org/10.4103/0019557X.99916

Shettar, M., Karkal, R., Kakunje, A., Mendonsa, R.D., Chandran, V.M., 2017. Facebook addiction and loneliness in the post-graduate students of a university in southern India. Int. J. Soc. Psychiatry 63, 325-329. https://doi.org/https://dx.doi.org/10.1177/0020764017705895

Singh, A., Mishra, N., 2009. Loneliness, depression and sociability in old age. Ind Psychiatry J 18, 51-55. https://doi.org/10.4103/0972

Susheela, P., Valsaraj, B.P., Savitha, 2018. Depression, perceived loneliness and partial functional impairment among older adults. J. Clin. Diagnostic Res. 12, VC01-VC05. https://doi.org/10.7860/JCDR/2018/34086.12096

Tiwari, S., 2013. Loneliness: A disease? Indian J. Psychiatry 55, 320. https://doi.org/10.4103/0019-5545.120536

Upmanyu, V.V., Sehgal, R., Upmanyu, S., 1994. Factor analytic study of personality correlates of loneliness among Indian university students. Psychol. Stud. (Mysore). 39, 28-33.

Wilson, G., Hill, M., Kiernan, M.D., 2018. Loneliness and social isolation of military veterans: systematic narrative review. Occup. Med. (Chic. Ill). 68, 600-609. https://doi.org/10.1093/occmed/kqy160

Yang, F., Gu, D., 2019. Predictors of loneliness incidence in Chinese older adults from a life course perspective: a national longitudinal study. Aging Ment. Health 1-10. https://doi.org/10.1080/13607863.2018.1558174

Yanguas, J., Pinazo-Henandis, S., Tarazona-Santabalbina, F.J., 2018. The complexity of 
loneliness. Acta Biomed. 89, 302-314. https://doi.org/10.23750/abm.v89i2.7404

Ybarra, M.L., Alexander, C., Mitchell, K.J., 2005. Depressive symptomatology, youth Internet use, and online interactions: A national survey. J. Adolesc. Heal. 36, 9-18. https://doi.org/10.1016/j.jadohealth.2003.10.012

Zhong, B.-L., Liu, X.-J., Chen, W.-C., Chiu, H.F.-K., Conwell, Y., 2018. Loneliness in Chinese older adults in primary care: prevalence and correlates. Psychogeriatrics 18, 334-342. https://doi.org/10.1111/psyg.12325 
Supplementary file: Quality assessment of the recruited studies (studies published from 1991 to 2013)

\begin{tabular}{|c|c|c|c|c|c|c|c|c|}
\hline S. No. & Evaluation criteria & $\begin{array}{l}\text { (Bhogle, } \\
\text { 1991) }\end{array}$ & $\begin{array}{l}\text { (Upmanyu } \\
\text { et al., 1994) }\end{array}$ & $\begin{array}{l}\text { (Singh and } \\
\text { Mishra, } \\
\text { 2009) }\end{array}$ & $\begin{array}{l}\text { (Nayyar } \\
\text { and Singh, } \\
\text { 2011) }\end{array}$ & $\begin{array}{l}\text { (Samanta et } \\
\text { al., 2012) }\end{array}$ & $\begin{array}{l}\text { (Mishra et } \\
\text { al., 2013) }\end{array}$ & $\begin{array}{l}\text { (Ninan and } \\
\text { Gopinath, } \\
\text { 2013) }\end{array}$ \\
\hline 1 & $\begin{array}{l}\text { Was the research question or } \\
\text { objective in this paper clearly stated? }\end{array}$ & Yes & Yes & Yes & Yes & Yes & Yes & Yes \\
\hline 2 & $\begin{array}{l}\text { Was the study population clearly } \\
\text { specified and defined? }\end{array}$ & Yes & Yes & Yes & Yes & Yes & Yes & Yes \\
\hline 3 & $\begin{array}{l}\text { Was the participation rate of eligible } \\
\text { persons at least } 50 \% ?\end{array}$ & NR & NR & NR & NR & Yes & Yes & NR \\
\hline 4 & $\begin{array}{l}\text { Were all the subjects selected or } \\
\text { recruited from the same or similar } \\
\text { populations (including the same time } \\
\text { period)? Were inclusion and } \\
\text { exclusion criteria for being in the } \\
\text { study prespecified and applied } \\
\text { uniformly to all participants? }\end{array}$ & Yes & Yes & Yes & Yes & Yes & Yes & Yes \\
\hline 5 & $\begin{array}{l}\text { Was a sample size justification, } \\
\text { power description, or variance and } \\
\text { effect estimates provided? }\end{array}$ & No & No & No & No & No & Yes & No \\
\hline 6 & $\begin{array}{l}\text { For the analyses in this paper, were } \\
\text { the exposure(s) of interest measured } \\
\text { prior to the outcome(s) being } \\
\text { measured? }\end{array}$ & NA & NA & NA & NA & NA & NA & NA \\
\hline 7 & $\begin{array}{l}\text { Was the timeframe sufficient so that } \\
\text { one could reasonably expect to see an } \\
\text { association between exposure and } \\
\text { outcome if it existed? }\end{array}$ & NA & NA & NA & $\mathrm{NA}$ & NA & NA & NA \\
\hline
\end{tabular}




\begin{tabular}{|c|c|c|c|c|c|c|c|c|}
\hline 8 & $\begin{array}{l}\text { For exposures that can vary in } \\
\text { amount or level, did the study } \\
\text { examine different levels of the } \\
\text { exposure as related to the outcome } \\
\text { (e.g., categories of exposure, or } \\
\text { exposure measured as continuous } \\
\text { variable)? }\end{array}$ & Yes & No & No & No & No & Yes & No \\
\hline 9 & $\begin{array}{l}\text { Were the exposure measures } \\
\text { (independent variables) clearly } \\
\text { defined, valid, reliable, and } \\
\text { implemented consistently across all } \\
\text { study participants? }\end{array}$ & Yes & Yes & No & Yes & Yes & Yes & Yes \\
\hline 10 & $\begin{array}{l}\text { Was the exposure(s) assessed more } \\
\text { than once over time? }\end{array}$ & No & No & No & No & No & No & No \\
\hline 11 & $\begin{array}{l}\text { Were the outcome measures } \\
\text { (dependent variables) clearly defined, } \\
\text { valid, reliable, and implemented } \\
\text { consistently across all study } \\
\text { participants? }\end{array}$ & Yes & Yes & Yes & Yes & Yes & Yes & Yes \\
\hline 12 & $\begin{array}{l}\text { Were the outcome assessors blinded } \\
\text { to the exposure status of participants? }\end{array}$ & NA & NA & NA & NA & NA & NA & NA \\
\hline 13 & $\begin{array}{l}\text { Was loss to follow-up after baseline } \\
20 \% \text { or less? }\end{array}$ & NA & NA & NA & NA & NA & NA & NA \\
\hline \multirow[t]{2}{*}{14} & $\begin{array}{l}\text { Were key potential confounding } \\
\text { variables measured and adjusted } \\
\text { statistically for their impact on the } \\
\text { relationship between exposure(s) and } \\
\text { outcome(s)? }\end{array}$ & No & No & No & No & No & No & No \\
\hline & Overall quality rating & Fair & Fair & Poor & Fair & Fair & Good & Fair \\
\hline
\end{tabular}


Supplementary file-continued (studies published from 2016 to 2019)

\begin{tabular}{|c|c|c|c|c|c|c|c|}
\hline S. No. & Evaluation criteria & $\begin{array}{l}\text { (Anil et al., } \\
2016)\end{array}$ & $\begin{array}{l}\text { (Shettar et } \\
\text { al., 2017) }\end{array}$ & $\begin{array}{l}\text { (Susheela et } \\
\text { al., 2018) }\end{array}$ & $\begin{array}{l}\text { (Grover et al., } \\
\text { 2018) }\end{array}$ & $\begin{array}{l}\text { (Malhotra et al., } \\
\text { 2019) }\end{array}$ & $\begin{array}{l}\text { (Grover et al., } \\
\text { 2019) }\end{array}$ \\
\hline 1 & $\begin{array}{l}\text { Was the research question or } \\
\text { objective in this paper clearly stated? }\end{array}$ & Yes & Yes & Yes & Yes & Yes & Yes \\
\hline 2 & $\begin{array}{l}\text { Was the study population clearly } \\
\text { specified and defined? }\end{array}$ & Yes & Yes & Yes & Yes & Yes & Yes \\
\hline 3 & $\begin{array}{l}\text { Was the participation rate of eligible } \\
\text { persons at least } 50 \% \text { ? }\end{array}$ & NR & NR & Yes & NR & Yes & Yes \\
\hline 4 & $\begin{array}{l}\text { Were all the subjects selected or } \\
\text { recruited from the same or similar } \\
\text { populations (including the same time } \\
\text { period)? Were inclusion and } \\
\text { exclusion criteria for being in the } \\
\text { study prespecified and applied } \\
\text { uniformly to all participants? }\end{array}$ & Yes & Yes & Yes & Yes & Yes & Yes \\
\hline 5 & $\begin{array}{l}\text { Was a sample size justification, } \\
\text { power description, or variance and } \\
\text { effect estimates provided? }\end{array}$ & Yes & No & Yes & No & Yes & Yes \\
\hline 6 & $\begin{array}{l}\text { For the analyses in this paper, were } \\
\text { the exposure(s) of interest measured } \\
\text { prior to the outcome(s) being } \\
\text { measured? }\end{array}$ & NA & NA & NA & NA & NA & NA \\
\hline 7 & $\begin{array}{l}\text { Was the timeframe sufficient so that } \\
\text { one could reasonably expect to see an } \\
\text { association between exposure and } \\
\text { outcome if it existed? }\end{array}$ & NA & NA & NA & NA & NA & NA \\
\hline
\end{tabular}




\begin{tabular}{|c|c|c|c|c|c|c|c|}
\hline 8 & $\begin{array}{l}\text { For exposures that can vary in } \\
\text { amount or level, did the study } \\
\text { examine different levels of the } \\
\text { exposure as related to the outcome } \\
\text { (e.g., categories of exposure, or } \\
\text { exposure measured as continuous } \\
\text { variable)? }\end{array}$ & Yes & Yes & Yes & Yes & Yes & Yes \\
\hline 9 & $\begin{array}{l}\text { Were the exposure measures } \\
\text { (independent variables) clearly } \\
\text { defined, valid, reliable, and } \\
\text { implemented consistently across all } \\
\text { study participants? }\end{array}$ & Yes & Yes & Yes & Yes & Yes & Yes \\
\hline 10 & $\begin{array}{l}\text { Was the exposure(s) assessed more } \\
\text { than once over time? }\end{array}$ & No & No & No & No & No & No \\
\hline 11 & $\begin{array}{l}\text { Were the outcome measures } \\
\text { (dependent variables) clearly defined, } \\
\text { valid, reliable, and implemented } \\
\text { consistently across all study } \\
\text { participants? }\end{array}$ & Yes & Yes & Yes & Yes & Yes & Yes \\
\hline 12 & $\begin{array}{l}\text { Were the outcome assessors blinded } \\
\text { to the exposure status of participants? }\end{array}$ & NA & $\mathrm{NA}$ & NA & $\mathrm{NA}$ & NA & NA \\
\hline 13 & $\begin{array}{l}\text { Was loss to follow-up after baseline } \\
20 \% \text { or less? }\end{array}$ & NA & NA & NA & NA & NA & NA \\
\hline \multirow[t]{2}{*}{14} & $\begin{array}{l}\text { Were key potential confounding } \\
\text { variables measured and adjusted } \\
\text { statistically for their impact on the } \\
\text { relationship between exposure(s) and } \\
\text { outcome(s)? }\end{array}$ & No & No & No & No & Yes & Yes \\
\hline & Overall quality rating & Good & Fair & Good & Fair & Good & Good \\
\hline
\end{tabular}

Abbreviations: NA- Not applicable, NR- Not reported. 